\title{
Controlling reaction rate of phase transfer hydrogenation of acetophenone by application of low external electric field
}

\author{
Nan Wang ${ }^{1}$, Alan Allgeier ${ }^{1}$, and Laurence Weatherley ${ }^{1}$ \\ ${ }^{1}$ University of Kansas
}

June 12, 2020

\begin{abstract}
The controllable mass transfer and reaction rate for phase transfer hydrogenation of acetophenone across a well-defined boundary were investigated. The effect of solvent was found important and 1-butanol exhibited the best performance among the five investigated homologous alcohol solvents, consistent with its higher solubility in water and greater dielectric constant. Initial reaction rates increased with increasing electric potential, consistent with enhanced mass transfer across the aqueous/organic boundary. At longer reaction times deactivation was apparent. It correlated with increasing voltage and is ascribed to lower equilibrium concentration of reactive species at the interface. External control over reaction rate was demonstrated by switching the applied electric potential over the course of the reaction. Effects of external electric field on enantioselectivity were also explored with reversal field direction. The changes correlate with catalyst decomposition.
\end{abstract}

\section{Introduction}

Controlling reaction rate and selectivity is a long-standing goal and challenge in chemistry. ${ }^{1-3}$ Conventional ways to control chemical reactions include heating, ${ }^{4}$ stirring, using solvents ${ }^{5}$ or catalysts. ${ }^{6}$ Recently, inspired by the pioneering work of Shaik and co-workers ${ }^{7-9}$ attention has turned to the manipulation of activation energy barriers and the stabilization of reactive species in chemical reactions by oriented external electric fields (OEEFs). ${ }^{10,11}$ The influence of OEEFs upon chemical reaction performance has raised a number of scientifically interesting questions, and increasing evidence has shown that OEEFs could be employed as smart reagents and catalysts in chemistry. ${ }^{10,12-15}$ It was proposed by Meir et al. ${ }^{8}$ that organic reactions can be theoretically accelerated when an external electric field is oriented along the "reaction axis" which enhances the electron flow, leading to a lower reaction barrier and the stabilization of transition states. This simulated prediction was experimentally demonstrated by Aragones et al. in an OEEF promoted Diels-Alder reaction at the single molecule scale. ${ }^{16}$ Huang et al. also proved this idea by selective catalysis of a Diel-Alder addition reaction with an external electric field oriented along the reaction coordinate. ${ }^{17}$ More importantly, Wang et al. predicted that the reaction enantioselectivity may be controlled by aligning the electric field along the dipole moment of the reactants. ${ }^{2}$ While it is potentially significant for asymmetric synthesis, there is no direct experimental evidence so far to support it.

Despite the promising prospects, there are two major challenges to be met in order to utilize OEEFs for controlling reaction rates and selectivity. One requirement is to orient the electric field along the reaction axis, ${ }^{10}$ the other is the high electric field strength $\left(10^{7}\right.$ to $\left.10^{9} \mathrm{~V} / \mathrm{m}\right) \cdot{ }^{17,18}$ At molecular level, these challenges can be addressed by using scanning tunneling microscope break junction (STMBJ) techniques ${ }^{16,19,20}$ or mechanically controllable break junction (MCBJ) techniques. ${ }^{17,21,22}$ Nevertheless, when conducting experiments on a bulk scale, the question of how to align the electric field along the reaction axis arises when thousands of reactant molecules are randomly oriented in the reaction system. Furthermore, applying thousands of volts to a lab-scale (or even larger scales) reactor could pose safety issues. Therefore, the significance of OEEFs on controlling reaction rates and selectivity experimentally in bulk quantity reactions for organic synthesis 
remains understudied. However, apart from lowering the reaction barrier with huge energy input, a simple way to manipulate the reaction rates by external electric fields is to control the diffusion of reactive species in the reaction system, ${ }^{23}$ and alter the local concentration of reactants. ${ }^{24}$ This has been validated in our previous study that the reaction rate can be either promoted or inhibited by simply flipping the orientation of the external electric field. ${ }^{25}$

To further explore the possibilities of controlling reaction rate and even selectivity with external electric field on bulk scales, catalytic transfer hydrogenation of acetophenone was studied in a biphasic liquid system. Previous studies showed that the phase transfer hydrogenation of acetophenone with sodium formate as hydrogen source is a mass transfer limited reaction happening at the organic-aqueous interface. ${ }^{25,26}$ When an external electric field was applied, the charged reactive species could be either transferred to the interface to promote the reaction or constrained in the corresponding liquid phases decelerating the reaction by the electrostatic forces. ${ }^{25}$ This raises the possibility of manipulating the reaction rates by controlling the diffusion of reactive species in the presence of varying electric field potential for interfacial reaction/catalysis in a biphasic liquid system.

In this contribution, we have demonstrated the ability of external electric fields to control reaction rates in the catalytic phase transfer hydrogenation of acetophenone and its influence on reaction enantioselectivity. Mathematical modeling and simulation results further support the controllable migration of reactive species under external electric fields. While evincing fundamental principles, the results also show the possible decomposition effects of the external electric field on the catalyst when applied in the negative orientation, resulting in lower reaction conversion and enantioselectivity. In general, this work shows the importance of comprehensively understanding the role of external electric field in organic synthesis and catalysis on bulk scale.

\section{Experimental section}

\subsection{Materials}

All chemicals used in this work were purchased from commercial reagent suppliers and used without further purification: acetophenone (Sigma-Aldrich, 99\%), sodium formate (Sigma-Aldrich, $99.0 \%), \operatorname{RuCl}(\mathrm{p}-$ cymene)[(S,S)-Ts-DPEN] (Sigma-Aldrich), 1-butanol (Sigma-Aldrich, 99.8\%), 1-pentanol (Sigma-Aldrich, [?] 99\%), 1-hexanol (Alfa Aesar, 99\%), 1-heptanol (Sigma-Aldrich, 98\%), 1-octanol (Sigma-Aldrich, [?] $99 \%)$.

\subsection{Experimental apparatus and procedure}

The phase transfer hydrogenation of acetophenone to produce 1-phenylethanol was studied in a specially made hollow cylindrical glass reactor with two ports for electrodes on the ends. The inner diameter of the reactor is about $20 \mathrm{~mm}$. Details of the experimental unit may be found elsewhere. ${ }^{25}$ Briefly, the biphasic system was set up with a sodium formate/water solution as the more dense aqueous phase and acetophenone/organic solvents as the less dense organic phase in which the Ru complex ( $\mathrm{RuCl}(\mathrm{p}$-cymene) $[(\mathrm{S}, \mathrm{S})-\mathrm{Ts}-\mathrm{DPEN}])$ was dissolved. The organic solvents used in conjunction with acetophenone were 1-butanol, 1-pentanol, 1-hexanol, 1-heptanol, and 1-octanol. An external electric field (DC voltage) from a high voltage power supply (PASCO SF-9585A) was applied across the two phases without agitation. Stainless steel or titanium electrodes were used. In a single experimental study, $0.34 \mathrm{~g}$ of sodium formate was fully dissolved in $1.5 \mathrm{~mL}$ of deionized water, while $117 \mu \mathrm{L}$ of acetophenone was mixed with $1.5 \mathrm{~mL}$ of organic solvent to dissolve $0.0064 \mathrm{~g} \mathrm{Ru}$ catalyst. The reactions were conducted at room temperature and pressure with positive or negative electric field applied during reaction. The detailed orientations of the electric field have been described previously. ${ }^{25}$ A positive orientation in this work is defined as the direction of the electric field when the anode is placed in the organic phase with the cathode in the aqueous phase. The conversion of acetophenone, total yield of 1-phenylethanol, and enantioselectivity of (S)-1-phenylethanol were determined by gas chromatography analysis.

2.3 Gas chromatography (GC) analysis 
$10 \mu \mathrm{L}$ of liquid samples from the organic phase were withdrawn after each reaction and diluted to $1.5 \mathrm{~mL}$ with methanol for gas chromatography analysis. An Agilent $6890 \mathrm{~N}$ gas chromatograph equipped with a CP-Chirasil-Dex CB column $(25 \mathrm{~m} \times 0.25 \mathrm{~mm})$ and a flame ionization detector was used to identify reaction products and determine enantioselectivity under the following conditions: helium as carrier gas $(3 \mathrm{~mL} / \mathrm{min})$, inlet and detector temperature $250{ }^{\circ} \mathrm{C}$, oven temperature $115^{\circ} \mathrm{C}$.

\subsection{Nuclear Magnetic Resonance (NMR) spectroscopy analysis}

Four samples were prepared for NMR analysis. Sample 1 was prepared by dissolving $5.8 \mathrm{mg}$ of $\mathrm{RuCl}(\mathrm{p}-$ cymene) $[(\mathrm{S}, \mathrm{S})-\mathrm{Ts}-\mathrm{DPEN}]$ in $500 \mu \mathrm{L}$ of $\mathrm{MeOH}-\mathrm{d} 4$ (Cambridge Isotopes). Sample 2 was obtained by dissolving $6.4 \mathrm{mg}$ of $\mathrm{RuCl}(\mathrm{p}$-cymene)[(S,S)-Ts-DPEN] in a mixture of $1.5 \mathrm{~mL}$ 1-butanol and $117 \mu \mathrm{L}$ acetophenone. Samples 3 and 4 were collected from reactions under positive and negative $15 \mathrm{~V}$ of electric potential respectively after $24 \mathrm{~h}$ with titanium electrodes and 1-butanol as solvent. $500 \mu \mathrm{L}$ of each liquid sample were pipetted into a $5 \mathrm{~mm}$ NMR tube (Wilmad Lab-Glass, Vineland, NJ). All NMR spectra were acquired on a $500 \mathrm{MHz}$ Bruker AVIII spectrometer equipped with a cryogenically cooled X-channel observe probe. All data was analyzed using MestreNova NMR software (Santiago De Compostela, Spain).

\subsection{X-ray florescence (XRF) analysis}

A handheld XRF analyzer (Olympus, Delta Professional) with Ru anode and silicon drift detector was used for fast determination of the metal contents in the degradation products of stainless-steel electrode. These solid samples were collected from the interface after reaction and dried prior for analysis. Precious metal mode with a targeted collimator was employed during analysis. All samples were scanned three times and analyzed with the workstation setup.

\section{Theoretical modeling and simulation}

The transport of the charged reactive species, referred to as electromigration in the present study, was modeled based on the Nernst-Plank equation. ${ }^{27-29}$ The molar flux of species $i, J_{i}$, is expressed in Equation (1), describing the diffusion by concentration difference and migration by electrostatic force. The convection term is not included due to the fact that the experiments were conducted in a quiescent hydrodynamic environment.

$J_{i}=-D_{i} \nabla c_{i}-\frac{D_{i} z_{i} F}{\mathrm{RT}} c_{i} \nabla \Phi(1)$

where $D_{i}$ is the diffusion coefficient $\left(\mathrm{m}^{2} / \mathrm{s}\right), c_{i}$ is the ion concentration $\left(\mathrm{mol} / \mathrm{m}^{3}\right), z_{i}$ is the valence number, $F$ is the Faraday constant, $R$ is the gas constant, $T$ is the absolute temperature $(\mathrm{K})$, and $\Phi$ is the electric potential $(V)$. The Wilke-Chang equation was applied to estimate the mutual diffusion coefficient if necessary. ${ }^{30}$

The electric field in the domain is described by the Poisson differential equation ${ }^{30}$ :

$\nabla \bullet\left(\varepsilon_{0} \varepsilon_{r} \nabla \Phi\right)=-\rho_{v}(2)$

where $\epsilon_{0}$ is the dielectric constant of the free space, $\epsilon_{\rho}$ is the relative permittivity of materials, $\rho$ is the electric space charge density.

The equations were solved using finite element method in COMSOL Multiphysics 5.4. Simulation was conducted to understand the mass transfer of reactive species in the proposed reaction system, assuming no reaction nor flux at/across the interface. Concentration profiles of Ru catalyst and formate at the interface were calculated under various external electric fields.

\section{Results and discussion}

\subsection{Solvent effects}

The effects of the different organic solvents used in conjunction with the acetophenone reactant were first studied as their different physical and chemical properties could significantly affect the reaction performance, especially when an external electric field is applied. Five primary alcohols, namely 1-butanol, 1-pentanol, 1-hexanol, 1-heptanol, and 1-octanol as organic solvents were investigated due to their ability to maintain a 
stable biphasic reaction system. The experimental results are presented in Figure 1, showing total conversion values and product yields. Table 1 lists the physical and chemical properties of the five solvents. No corresponding aldehyde by-products were detected by GC analysis after the reactions, which confirms the role of sodium formate as hydrogen source and the concept of phase transfer hydrogenation. As we can see, addition of 1-butanol resulted in the best performance on promoting the transfer hydrogenation of acetophenone with conversion of $84.7 \%$ and yield of $76.8 \%$ after $24 \mathrm{~h}$ of reaction under $15 \mathrm{~V}$ of positive DC voltage with stainless-steel electrodes. The conversion and yield decreased gradually with the increase of the molecular weight of these homologous alcohol solvents.

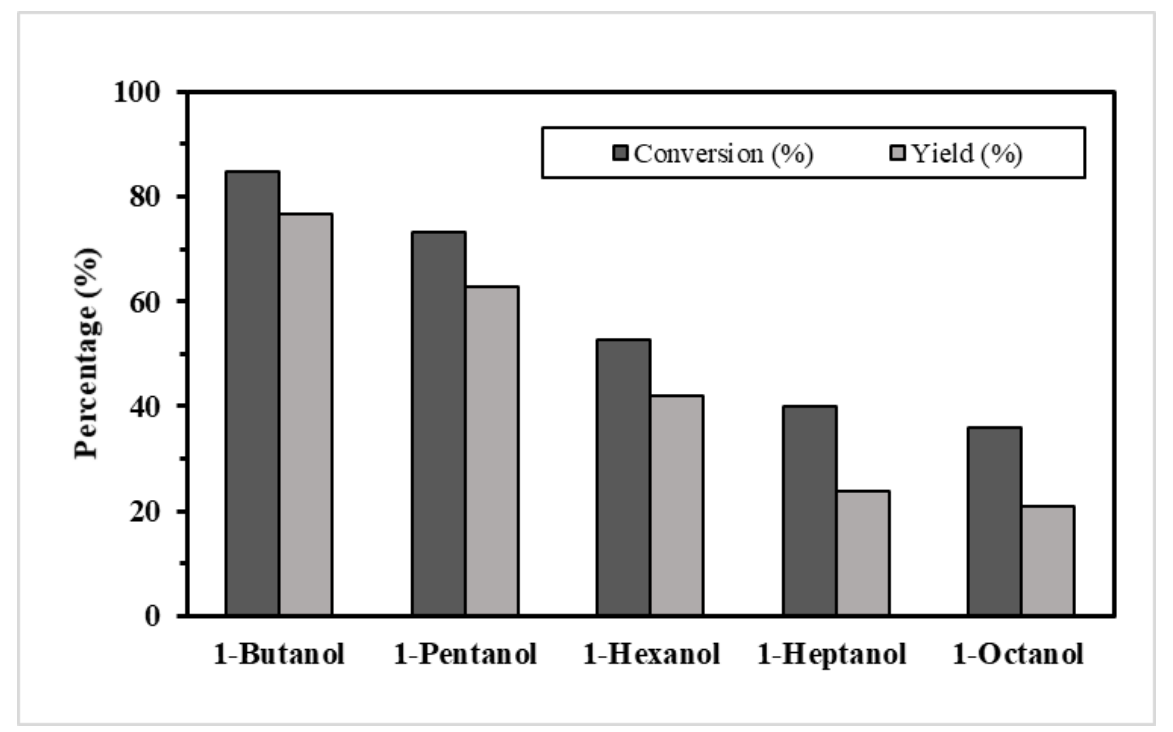

Figure 1. Acetophenone conversion (mol\%) and 1-phenelethanol yields (mol\%) under $15 \mathrm{~V}$ of positive external electric field for $24 \mathrm{~h}$ with different organic solvents and stainless-steel electrodes.

One possible reason for the significantly different performance may be their respective solubilities in water. Compared to the other solvents, 1-butanol has the highest solubility in water, which initially promotes most of the interaction between the reactants at the interface, resulting in the highest conversion and reaction rate (more than 2 fold faster than that with 1-octanol as solvent). On the other hand, these alcohols are protic solvents, which possess the ability to generate protons by dissociation. With the largest dielectric constant among the studied solvents, 1-butanol has the greatest potential to polarize and generate protons under an external electric field. These protons could further promote the dissociation of the neutral $\mathrm{Ru}$ compound ( $\mathrm{RuCl}(\mathrm{p}$-cymene)[(S-S)-Ts-DPEN]) into the cationic Ru complex (species 2 in Scheme 1) by removing the chloride ion, ${ }^{31,32}$ therefore accelerating and promoting the generation of the catalysts and ultimately increasing the production rate. While the dipole moment and electrical conductivity might be important in electrostatically promoted reactions according to our previous investigation ${ }^{25}$ and Shaik and coworkers, ${ }^{2}$ the differences of these properties between the five solvents are small. Accordingly, it is difficult to attribute the differences in reaction performance to dipole moment and conductivity in the present study.

Table 1. Properties of the studied organic solvents.

\begin{tabular}{lllll}
\hline Solvent & Electrical conductivity $(\mathrm{ns} / \mathrm{cm})$ & Solubility in water $\left(25^{\circ} \mathrm{C} / \text { mass } \%\right)^{33}$ & Dipole $(\mathrm{D})^{33}$ & Dielectric constant $\left(20^{\circ}\right.$ \\
\hline 1-butanol & $36^{34,35}$ & 7.3 & 1.66 & 17.84 \\
1-pentanol & $41^{34,35}$ & 2.14 & 1.7 & $15.13^{\mathrm{a}}$ \\
1-hexanol & - & 0.59 & - & 13.03 \\
1-heptanol & - & 0.164 & - & 11.75
\end{tabular}




\begin{tabular}{lllll}
\hline Solvent & Electrical conductivity $(\mathrm{ns} / \mathrm{cm})$ & Solubility in water $\left(25^{\circ} \mathrm{C} / \text { mass } \%\right)^{33}$ & Dipole $(\mathrm{D})^{33}$ & Dielectric constant $\left(20^{\circ}\right.$ \\
\hline 1-octanol & $140^{36}$ & 0.046 & 1.76 & 10.3 \\
\hline
\end{tabular}

a: This value was obtained at $25^{\circ} \mathrm{C}$.

\subsection{Electric field potential}

Various values of positive external electric field potential were applied to determine its effects on mass transfer and kinetics in the proposed reaction system. The conversion of acetophenone under different positive external electric field strengths, recorded at different times are shown in Figure 2. The data in this Figure are only for the acetophenone/1-butanol mixture using stainless steel electrodes. It was observed that the initial reaction rates increased with the increase of the electric potential. The conversion of acetophenone in the presence of an applied voltage of $100 \mathrm{~V}$ was observed to reach $44.5 \%$ after a reaction time of 2 hours, which is nearly 4 fold of the conversion attained at $15 \mathrm{~V}$. However, at longer reaction times, the rate of increase was observed to decline. The decline was greater at the higher voltages. For example, after $8 \mathrm{~h}$, a higher conversion was achieved at $15 \mathrm{~V}$ compared to the values observed at $30 \mathrm{~V}$ and $50 \mathrm{~V}$. The total conversion at $15 \mathrm{~V}$ reached to $84.7 \%$ after $24 \mathrm{~h}$ which is about $20 \%$ higher than that at $30 \mathrm{~V}$ and $40 \%$ higher than that at $50 \mathrm{~V}$.

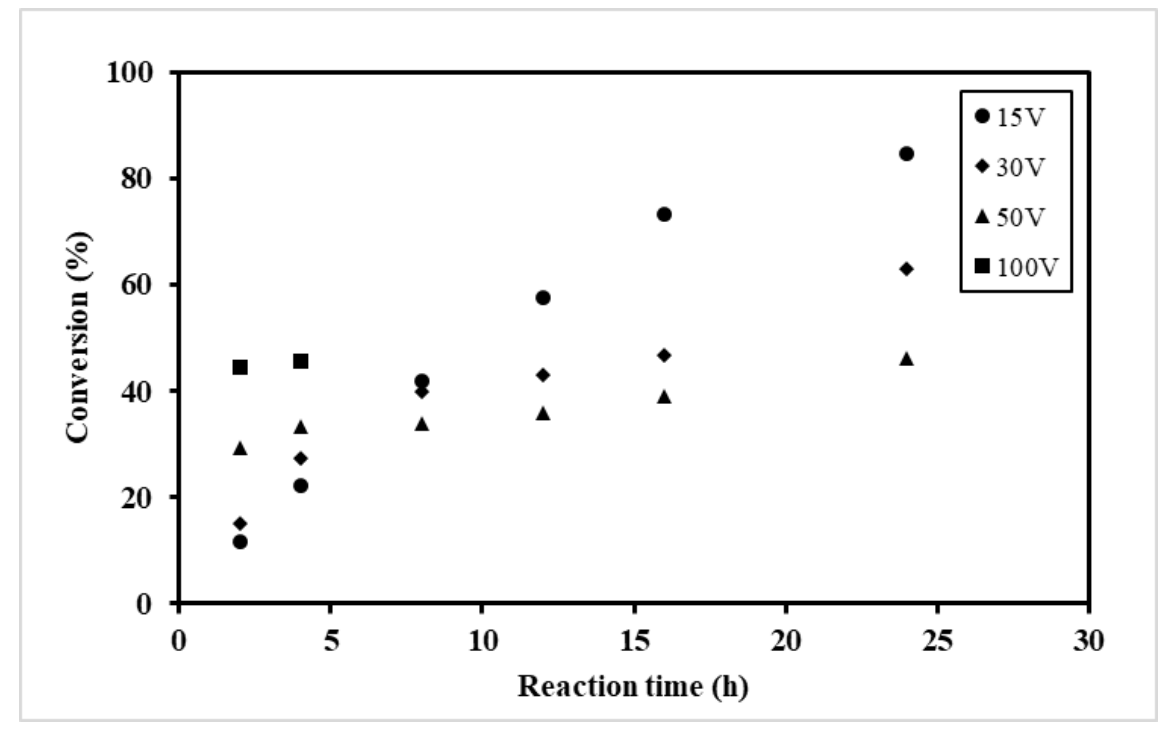

Figure 2. Acetophenone conversion (mol\%) under different positive external electric field for $24 \mathrm{~h}$ with stainless-steel electrodes and 1-butanol as solvent.

The increase in conversion with increased electric potential in the first few hours is consistent with the role of external electric field in controlling the reaction rates of the phase transfer hydrogenation reaction by controlling mass transfer of the charged reactive species. This is further supported by the simulation results in Figure 3. As we can see, the rates of electromigration of both formate and Ru catalyst were increased with the increase of voltage at the beginning. The enhancement of reaction rate observed at higher electric field is consistent with accelerated rates of transport of both formate anions and the cationic Ru catalysts to the liquid-liquid interface, resulting in more rapid reaction compared to that under lower electric potential. The simulation results also suggested that the equilibrium concentration of $\mathrm{Ru}$ catalyst at the interface is lower at higher voltage, which explains the corresponding lower conversion at longer reaction times. Additionally, a negatively charged alkoxide intermediate could be formed during the reaction as suggested by Pavlova et al. ${ }^{37}$ As the reaction continues, a portion of these alkoxide anions could be attracted to the anode electrode and constrained in the region close to it, preventing the reactive species from interacting and retarding the 
reaction rates. This inhibition is greater when a greater electrostatic force is applied, leading to the lower reaction rate as observed at an elevated electric potential for longer reaction times.

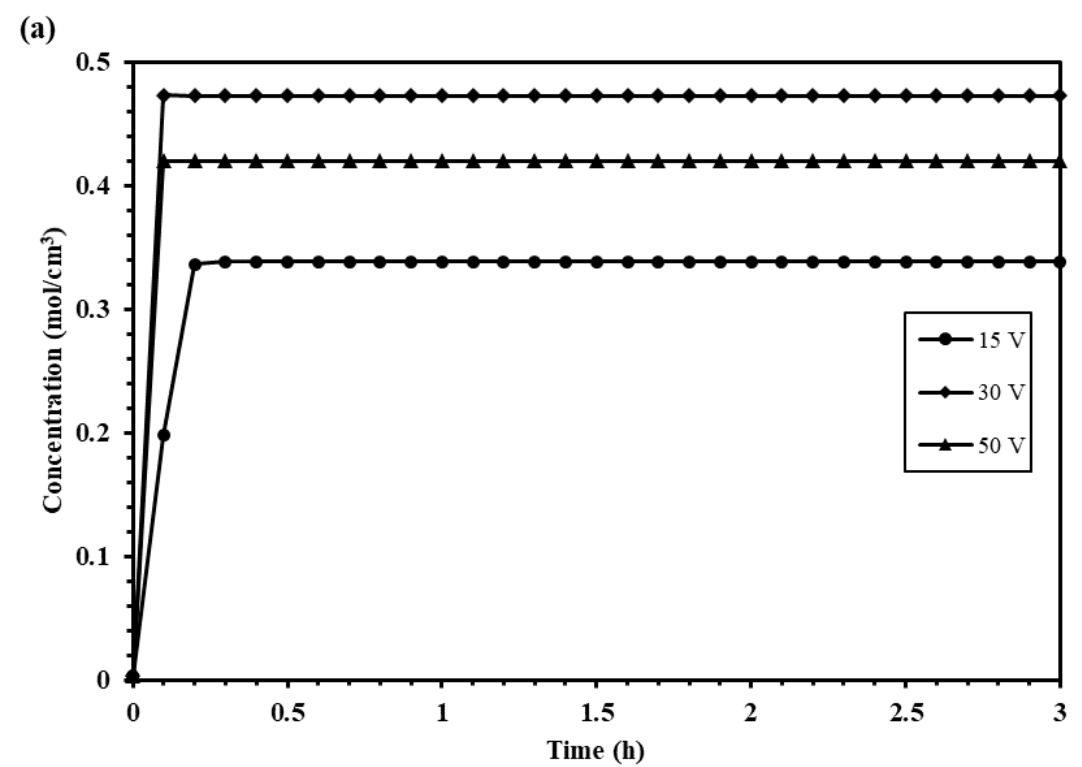

(b)

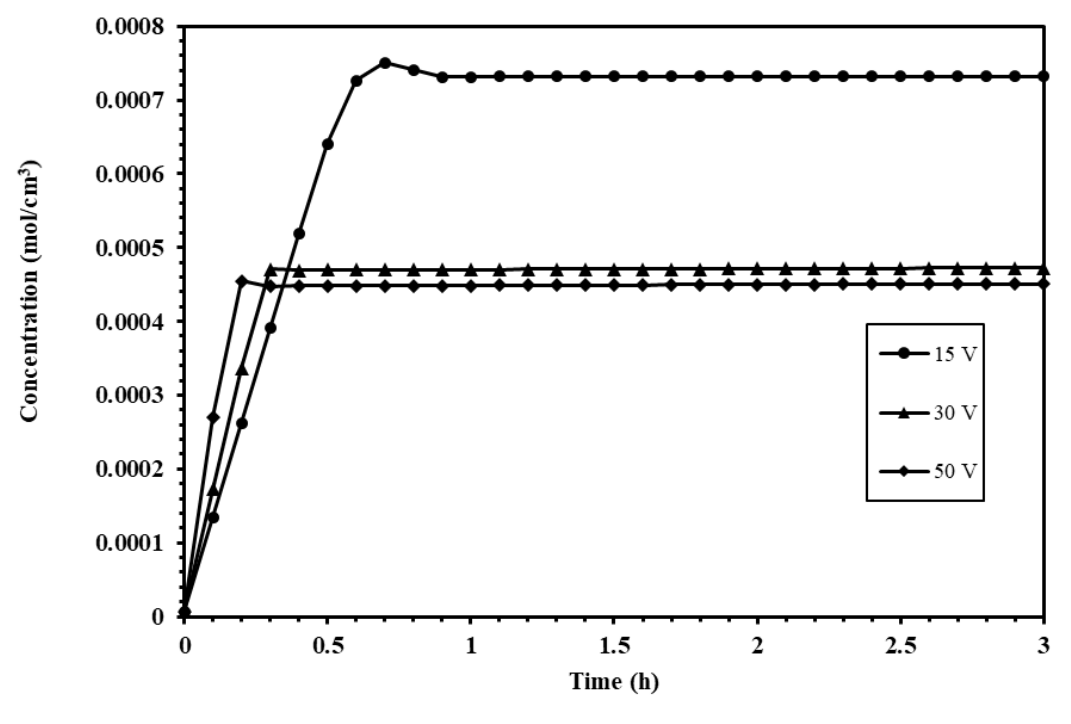

Figure 3. Simulated concentration profiles at the interface under various electric fields. (a) Concentration profile of formate ions, (b) concentration profile of $\mathrm{Ru}$ catalyst.

On the other hand, an external electric field induced current flow in the reaction system along with degradation of the anode electrode (immersed in the organic phase) were observed. Additionally, gas bubbles 
of unknown composition were generated on the cathode. The degradation of the electrode was confirmed by weight loss measurement (see Cases 1,2 and 3 in Table 2). The degradation products which formed a thin layer of black slurry at the liquid-liquid interface were characterized by XRF analysis. The analysis showed that the slurry consisted mainly of $\mathrm{Fe}, \mathrm{Ni}$, and $\mathrm{Cr}$ with a small amount of $\mathrm{Ru}$. For reactions under higher electric potentials, higher current flows and greater degradation rates of the electrode were observed as shown in Table 2 (Cases 1, 2, and 3). The decomposition of the electrode is consistent with galvanic corrosion, in which hydrogen is produced at the cathode with the corrosion occurring at the anode. At higher electric potentials, stronger galvanic corrosion effects would be expected, leading to greater electrode degradation. With more metal ions released from the degradation, higher current flows would be observed due to increased conductivity of the liquid phase. The degradation products formed at the interface could also contribute to the deceleration of the reaction by inhibiting mass transfer to the catalyst at the interface. At higher electric potential, a higher degradation was observed, leading to a higher blocking coverage of the interface and finally achieving a lower reaction rate and conversion.

To eliminate the negative effects of electrode degradation and clarify the inhibiting effects of high electric potential, chemically stable titanium electrodes were employed, and the results are recorded as Cases 4, 5, and 6 in Table 2, compared to those with stainless steel electrodes (Cases 1, 2, and 3). For experiments with different electric potential applied, the average degradation rate of titanium electrodes is less than $0.1 \% \mathrm{w} / \mathrm{w}$, and no apparent black slurry was observed. Although there was negligible evidence of electrode degradation of titanium electrodes, reaction performance showed the same trend as observed with stainless steel electrodes, when the applied electric potential was increased. This suggests that the actual electrode degradation plays a minimal role in changes in reaction performance, thus strongly suggesting the controllable migration of $\mathrm{Ru}$ catalyst and alkoxide ions under electrostatic force as being the main reason for the reaction deactivation and the importance of external electric field in controlling reaction rates. Furthermore, in spite of apparent reaction deactivation, a constant product ((S)-1-phenylethanol) enantioselectivity was achieved. This is further evidence that the deactivation is most likely due to the controllable migration of reactive species rather than catalyst decomposition.

A further set of experiments was conducted to investigate the reaction performance when the applied electrical potential was varied over the course of the reaction. With reference to Table 2, the data shown in the last three rows list the results of these experiments, labelled Cases 7, 8, and 9. In case 7, after reacting for $8 \mathrm{~h}$ under $30 \mathrm{~V}$, the electric potential was switched to $15 \mathrm{~V}$ for $16 \mathrm{~h}$, and the reaction yield reached to $67.5 \%$, which is an increase of $7.4 \%$ compared to that with solely $30 \mathrm{~V}$ applied for $24 \mathrm{~h}$ (Case 5). In case 8 and $9,30 \mathrm{~V}$ or $50 \mathrm{~V}$ were applied for $4 \mathrm{~h}$ respectively followed by $15 \mathrm{~V}$ applied for $20 \mathrm{~h}$. In these two cases, the yields of 1-phenylethanol increased when comparing to those with solely $30 \mathrm{~V}$ or $50 \mathrm{~V}$ applied for $24 \mathrm{~h}$. These results suggest that the reaction could be externally controlled by simply switching the applied electric potential over the course of the reaction.

Table 2. Summary for experiments under various positive external electric fields.

\begin{tabular}{llllllll}
\hline Case & Voltage $(\mathrm{V})$ & Time $(\mathrm{h})$ & Electrode & Current $(\mathrm{mA})$ & Weight loss $(\% \mathrm{w} / \mathrm{w})$ & Yield $(\%)$ & ee $(\%)$ \\
\hline 0 & 0 & 24 & - & $\mathrm{N} / \mathrm{A}$ & $\sim 0$ & 49.8 & 94.6 \\
1 & 15 & 24 & $\mathrm{SS}$ & 1.2 & 3.5 & 76.8 & 93.5 \\
2 & 30 & 24 & $\mathrm{SS}$ & 1.7 & 4.3 & 55.5 & 93.4 \\
3 & 50 & 24 & $\mathrm{SS}$ & 2.6 & 4.4 & 32.6 & 93.3 \\
4 & 15 & 24 & $\mathrm{Ti}$ & 0.2 & 0.085 & 67.4 & 94.5 \\
5 & 30 & 24 & $\mathrm{Ti}$ & 0.5 & 0.085 & 94.5 \\
6 & 50 & 24 & $\mathrm{Ti}$ & 1.6 & 0.09 & 26.6 & 94.5 \\
7 & $30-8 \mathrm{~h}+15-16 \mathrm{~h}$ & $30-8 \mathrm{~h}+15-16 \mathrm{~h}$ & $\mathrm{Ti}$ & 0.2 & 0.09 & 67.5 & 94.5 \\
8 & $30-4 \mathrm{~h}+15-20 \mathrm{~h}$ & $30-4 \mathrm{~h}+15-20 \mathrm{~h}$ & $\mathrm{Ti}$ & 0.2 & 0.02 & 63.9 & 94.3 \\
9 & $50-4 \mathrm{~h}+15-20 \mathrm{~h}$ & $50-4 \mathrm{~h}+15-20 \mathrm{~h}$ & $\mathrm{Ti}$ & 0.2 & 0.1 & 47.1 & 94.3 \\
\hline
\end{tabular}




\subsection{Proposed mechanism}

Based on the observations, a stepwise mechanism for the phase transfer hydrogenation of acetophenone with aqueous formate as hydrogen source and Ru-TsDPEN catalyst under an external electric field is proposed in Scheme 1. The cationic Ru complex $\mathbf{2}$ is generated by removal of chloride after compound $\mathbf{1}$ dissolves in 1-butanol. Following the formation, species $\mathbf{2}$ undergoes electromigration to the interface where a hydride is transferred to $\mathbf{2}$ from a formate to generate catalyst $\mathbf{3}$, with the release of $\mathrm{CO}_{2} \cdot{ }^{26}$ Instead of transferring both hydrogen to the substrate concertedly, ${ }^{31}$ only the hydride on $\mathrm{Ru}-\mathrm{H}$ in catalyst $\mathbf{3}$ is transferred to acetophenone with the proton on the $\mathrm{NH}_{2}$ moiety retained, ${ }^{37}$ resulting in the regeneration of species 2 and the formation of the corresponding alkoxide intermediate due to the stabilization of hydrogen bonding by water. ${ }^{37}$ Finally, the alkoxide picks up a proton from water to form the product, ${ }^{37}$ and the generated hydroxide further reacts with $\mathrm{CO}_{2}$ to form bicarbonate as observed in our previous study. ${ }^{25}$

\section{Hosted file}

image5.emf available at https://authorea.com/users/332910/articles/459244-controllingreaction-rate-of-phase-transfer-hydrogenation-of-acetophenone-by-application-of-lowexternal-electric-field

Scheme 1. Proposed mechanism for the phase transfer hydrogenation of acetophenone with aqueous formate as hydrogen source and Ru-TsDPEN catalyst.

The stoichiometry and rate expression consistent with the proposed mechanism are summarized in Equations (3) - (9), assuming that the coordination of formate and decarboxylation are reversible and equilibrated prior to hydrogen transfer, ${ }^{26}$ where $K_{1}$ is the equilibrium constant, and $k_{1}, k_{2}, k_{3}$ are the respective reaction rate constants. $A C P$ and $A C P H^{-}$represent acetophenone and its corresponding alkoxide, while 1- $P E$ refers to 1phenylethanol and $[R u]$ represents the total concentration of catalyst. $\mathrm{As}_{\mathrm{CO}_{2}}$ rapidly reacts with hydroxide during the reaction, the concentration of $\mathrm{CO}_{2}$ is expected to be small, therefore the rate expression can be further simplified as a second order kinetics over the concentration of catalyst and substrate (Equation (9)). This is consistent with the study by $\mathrm{Wu}$ et al. ${ }^{26}$ and with the above observation showing that the reaction rates were limited by the concentration of $\mathrm{Ru}$ catalyst and the alkoxide at the interface region. Both of these concentrations were affected by the electromigration under the influence of external electric fields. These observations suggest an important impact of electric fields on kinetics of interfacial reactions involving reactive ions.

$2+\mathrm{HCOO}^{-} 3+\mathrm{CO}_{2}(3)$
$3+\mathrm{ACP} 2+\mathrm{ACPH}^{-}(4)$

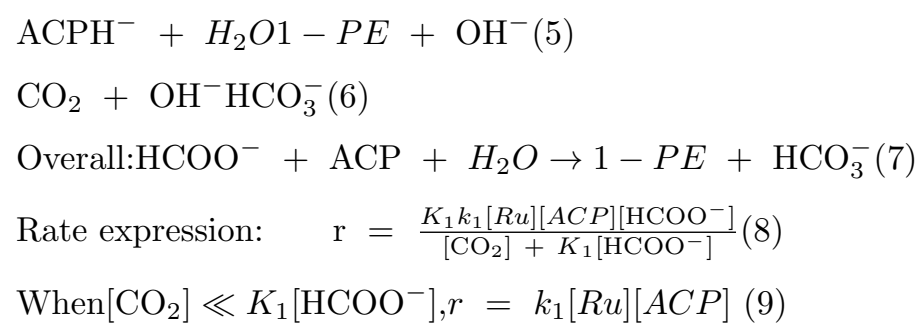

4.4 Electric field polarity and product enantioselectivity

As shown in Table 2, the enantioselectivity of (S)-1-phenylethanol remained constant with respect to electric field potential applied in the positive orientation. Since the orientation of OEEF was proposed as being important for controlling reaction selectivity, a reversed external electric field was then applied to the reaction system. The results are presented in Table 3. In these cases, the reaction was inhibited when the electric potential was applied in the negative orientation, which further confirms the hypothesis of controllable mass transfer and reaction rates by external electric fields, in accord with past findings. The yield of 1phenylethanol increased with the increased negative electric field strength, which was unexpected as a larger 
inhibiting electrostatic force would be expected to result in a lower reaction rate and conversion. This increase is likely due to the significant increase on the current flow (Figure 4) in the reaction system. Increase in current flow is consistent with the local temperature increase due to joule heating, which would compete with the inhibiting effects of higher negative electrostatic force and possibly promote the reaction.

Table 3. Summary for experiments with various negative external electric fields applied.

\begin{tabular}{lllllll}
\hline Voltage $(\mathrm{V})$ & Voltage $(\mathrm{V})$ & Time $(\mathrm{h})$ & Electrode & Current $(\mathrm{mA})$ & Yield $(\%)$ & EE $(\%)$ \\
\hline 0 & 0 & 24 & - & N/A & 49.8 & 94.6 \\
-5 & 24 & 24 & $\mathrm{Ti}$ & - & 16.9 & 91.0 \\
-10 & 24 & 24 & $\mathrm{Ti}$ & - & 22.4 & 86.0 \\
-15 & 24 & 24 & $\mathrm{Ti}$ & - & 23.0 & 66.1 \\
-20 & 24 & 24 & $\mathrm{Ti}$ & - & 45.6 & 82.9 \\
-30 & 24 & 24 & $\mathrm{Ti}$ & - & 48.4 & 75.2 \\
\hline
\end{tabular}

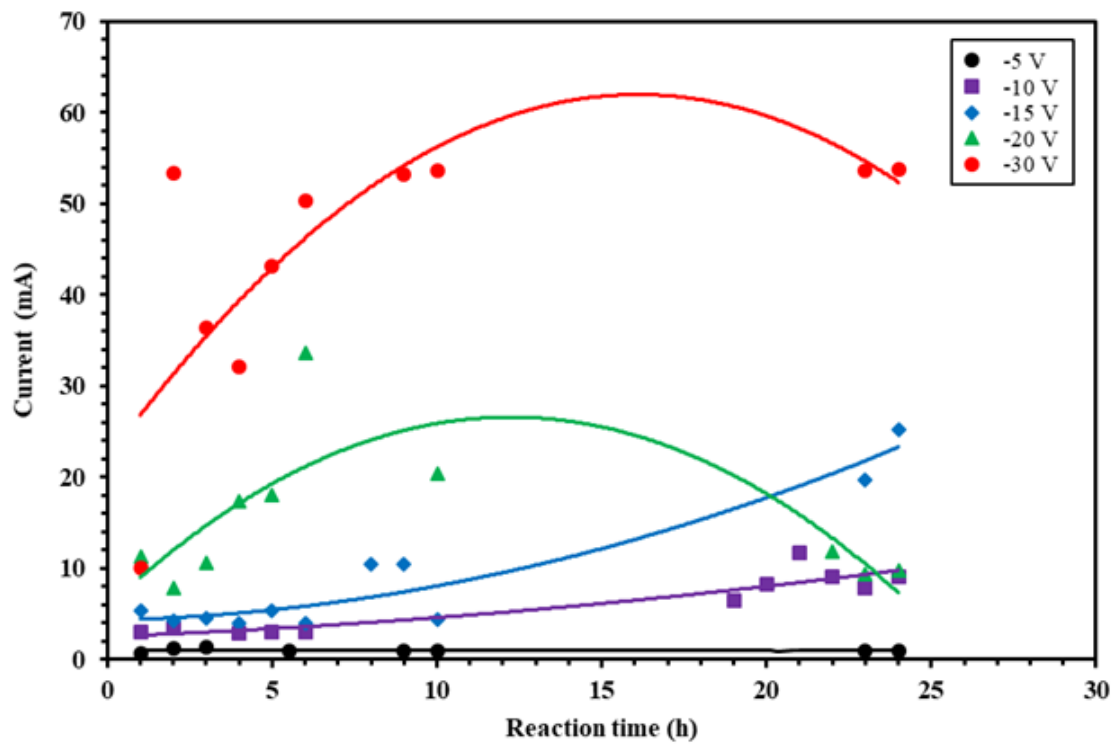

Figure 4. Current flow at different reaction time under various negative external electric fields.

On the other hand, with the increase of the inhibiting electric potential, decreased (S)-1-phenylethanol enantioselectivities were observed. However, these changes in the enantioselectivity are probably not due to the ability of OEEF to control reaction selectivity but are more likely attributed to the catalyst decomposition. Figure 5 showed the NMR analysis for the identification of the structures of the catalysts before and after reactions. Samples 1 (spectrum A) and 2 (spectrum B) were obtained by dissolving the Ru catalysts in $\mathrm{CD}_{3} \mathrm{OD}$ and reaction mixture (1-butanol and acetophenone mixture) respectively, and peaks from the Ru catalyst are clearly present at approximately 6.57 and $6.79 \mathrm{ppm}$. For sample 3 (spectra C), collected from the reaction run at positive $15 \mathrm{~V}$ for $24 \mathrm{~h}$, small signals (due to the low initial concentration and difficulty of detection in $1 \mathrm{H}$ NMR spectra) for the Ru catalyst can be seen. However, the NMR spectra for sample 4, from the reaction run at negative $15 \mathrm{~V}$ for $24 \mathrm{~h}$, showed no signals for the $\mathrm{Ru}$ catalyst. It remains unknown if the decomposition of the $\mathrm{Ru}$ catalyst was caused by the negative external electric field, and further investigation is required. 


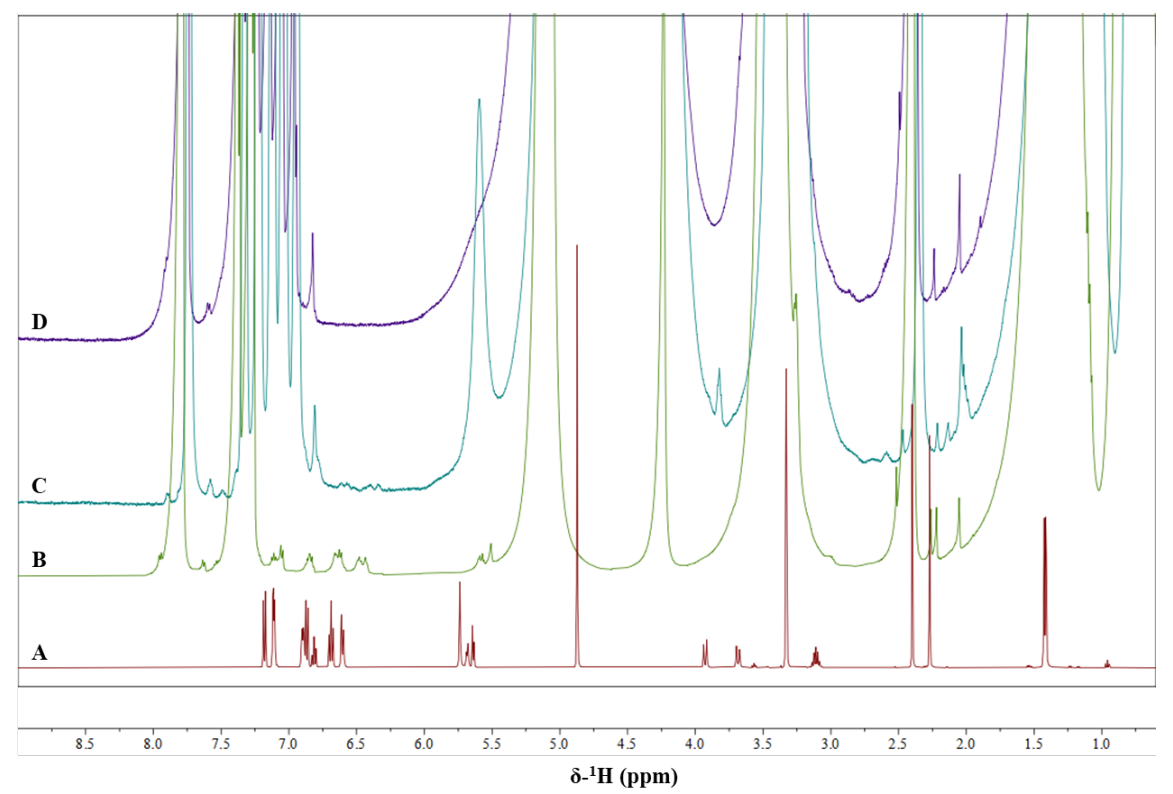

Figure 5. Stack plot of 1D 1H NMR spectra of samples 1 (A), 2 (B), 3 (C) and 4 (D). To highlight the minor species, spectra $\mathrm{B}$ was expanded vertically by 8 times, and spectra $\mathrm{C}$ and $\mathrm{D}$ were expanded by 24 times.

\section{Conclusion}

The reported work demonstrated experimentally controllable reaction rates by low voltage external electric fields for the catalytic transfer hydrogenation of acetophenone. The influence of applied electric potential on reaction performance was evaluated experimentally and the controllable mass transfer of reactive species by electric fields was supported by simulation results. Significant improvements were shown at low values of voltage relative to a control of zero volts, with an optimum voltage of positive $15 \mathrm{~V}$. Increases beyond this value to positive $30 \mathrm{~V}$ and $50 \mathrm{~V}$ showed significant reduction in performance due to lower equilibrium concentration of $\mathrm{Ru}$ catalyst at the interface and possible formation and migration of the alkoxide intermediate during reaction. The degradation products from the electrolytic corrosion of the stainless-steel electrodes did not significantly impact the hydrogenation reaction. The influence of different external electric fields on the concentrations of reactive species at the interface was suggested as the main reason for the differences in reaction performance. Enantiomeric excess values were measured, and no significant changes were observed with variation in the positive voltage values. A mechanism and reaction rate expression were proposed based on the observations, suggesting the importance of external electric fields in reactions involving reactive ions.

The direction of the applied electric field was proved to be important as both reaction conversion and enantioselectivity were significantly reduced when the electric field was in the negative orientation. A monotonic increase in conversion was observed as voltage increased from negative $5 \mathrm{~V}$ to negative $50 \mathrm{~V}$ due to possible temperature increase resulted from the increase of current. Catalyst decomposition by the influence of negative voltages was concluded as the main reason for reduced enantioselectivity, as implied from the NMR analysis.

Overall, this work extends the scope for controllable synthesis of organic reactions conducted in two phase liquid systems and raises an opportunity for green engineering with minimal energy consumption in the proposed reaction system. It is also important for understanding the influence of external electric fields on mass transfer and kinetics in biphasic liquid systems used for organic synthesis and interfacial catalysis on macroscale.

\section{Acknowledgements}


The work was supported by the Albert Learned Endowment from the University of Kansas. The authors also would like to acknowledge Justin T. Douglas for his assistance in the NMR analysis and Zachary R. Day for his assistance in the XRF analysis.

\section{References}

1. Jiang Y, Huan Q, Fabris L, Bazan GC, Ho W. Submolecular control, spectroscopy and imaging of bond-selective chemistry in single functionalized molecules. Nat Chem. 2013;5(1):36-41.

2. Wang Z, Danovich D, Ramanan R, Shaik S. Oriented-External electric fields create absolute enantioselectivity in Diels-Alder reactions: importance of the molecular dipole moment. JACS.2018;140(41):1335013359 .

3. Ordomsky VV, Khodakov AY, Legras B, Lancelot C. Fischer-Tropsch synthesis on a ruthenium catalyst in two-phase systems: an excellent opportunity for the control of reaction rate and selectivity. Catal Sci Technol. 2014;4(9):2896-2899.

4. Stuerga D, Gonon K, Lallemant M. Microwave heating as a new way to induce selectivity between competitive reactions. application to isomeric ratio control in sulfonation of naphthalene.Tetrahedron. 1993;49(28):6229-6234.

5. Burk MJ, Feng S, Gross MF, Tumas W. Asymmetric catalytic hydrogenation reactions in supercritical carbon dioxide. JACS.1995;117(31):8277-8278.

6. Lee I, Morales R, Albiter MA, Zaera F. Synthesis of heterogeneous catalysts with well shaped platinum particles to control reaction selectivity. PNAS. 2008;105(40):15241-15246.

7. Shaik S, de Visser SP, Kumar D. External electric field will control the selectivity of enzymatic-like bond activations. JACS.2004;126(37):11746-11749.

8. Meir R, Chen H, Lai W, Shaik S. Oriented electric fields accelerate Diels-Alder reactions and control the endo/exo selectivity. ChemPhysChem. 2010;11(1):301-310.

9. Hirao H, Chen H, Carvajal MA, Wang Y, Shaik S. Effect of external electric fields on the C-H bond activation reactivity of nonheme iron-oxo reagents. JACS. 2008;130(11):3319-3327.

10. Shaik S, Mandal D, Ramanan R. Oriented electric fields as future smart reagents in chemistry. Nat Chem.2016;8(12):1091-1098.

11. Shaik S, Ramanan R, Danovich D, Mandal D. Structure and reactivity/selectivity control by orientedexternal electric fields. Chem Soc Rev. 2018;47(14):5125-5145.

12. Ramanan R, Danovich D, Mandal D, Shaik S. Catalysis of methyl transfer reactions by oriented external electric fields: are gold-thiolate linkers innocent? JACS. 2018;140(12):4354-4362.

13. Che F, Gray JT, Ha S, Kruse N, Scott SL, McEwen J-S. Elucidating the roles of electric fields in catalysis: a perspective.ACS Catal. 2018;8(6):5153-5174.

14. Cassone G, Pietrucci F, Saija F, Guyot F, Saitta AM. One-step electric-field driven methane and formaldehyde synthesis from liquid methanol. Chem Sci. 2017;8(3):2329-2336.

15. Cassone G, Sponer J, Sponer JE, Pietrucci F, Saitta AM, Saija F. Synthesis of (d)-erythrose from glycolaldehyde aqueous solutions under electric field. Chem Commun.2018;54(26):3211-3214.

16. Aragonès AC, Haworth NL, Darwish N, et al. Electrostatic catalysis of a Diels-Alder reaction. Nature.2016;531(7592):88-91.

17. Huang X, Tang C, Li J, et al. Electric field-induced selective catalysis of single-molecule reaction. Sci Adv.2019;5(6):eaaw3072. 
18. Cassone G, Sofia A, Rinaldi G, Sponer J. Catalyst-free hydrogen synthesis from liquid ethanol: an ab initio molecular dynamics study. J Phys Chem C. 2019;123(14):9202-9208.

19. Xu B, Tao NJ. Measurement of single-molecule resistance by repeated formation of molecular junctions. Science.2003;301:1221-1223.

20. Venkataraman L, Klare JE, Nuckolls C, Hybertsen MS, Steigerwald ML. Dependence of single-molecule junction conductance on molecular conformation. Nature. 2006;442(7105):904-907.

21. Frisenda R, Janssen VAEC, Grozema FC, van der Zant HSJ, Renaud N. Mechanically controlled quantum interference in individual $\pi$-stacked dimers. Nat Chem. 2016;8(12):1099-1104.

22. Schwarz F, Kastlunger G, Lissel F, et al. Field-induced conductance switching by charge-state alternation in organometallic single-molecule junctions. Nat Nanotechnol. 2016;11(2):170-176.

23. Tachiya M. Effect of an external electric field on the rate of diffusion-controlled reactions. $J$ Chem Phys.1987;87(8):4622-4626.

24. Deshmukh SD, Tsori Y. Communication: control of chemical reactions using electric field gradients. $J$ Chem Phys.2016;144(19):191102.

25. Wang N, Kaminski K, Petera J, Allgeier AM, Weatherley LR. Electrostatically enhanced catalytic phase transfer hydrogenation of acetophenone under low external electric field. Chem Eng J.2019;374:1096-1101.

26. Wu X, Liu J, Di Tommaso D, et al. A multilateral mechanistic study into asymmetric transfer hydrogenation in water. Chem Eur J. 2008;14(25):7699-7715.

27. Bier M, Palusinski OA, Mosher RA, Saville DA. Electrophoresis: mathematical modeling and computer simulation.Science. 1983;219(4590):1281-1287.

28. Levine ML, Cabezas H, Bier M. Transport of solutes across aqueous phase interfaces by electropgoresis - mathematical modeling.J Chromatogr. 1992;607(1):113-118.

29. Zhu HT, Yang B, Gao CJ, Wu YQ. Ion transfer modeling based on Nernst-Planck theory for saline water desalination during electrodialysis process. Asia-Pac J Chem Eng. 2020;15(2):11.

30. Petera J, Weatherley LR, Rooney D, Kaminski K. A finite element model of enzymatically catalyzed hydrolysis in an electrostatic spray reactor. Comput Chem Eng. 2009;33(1):144-161.

31. Ohkuma T, Utsumi N, Tsutsumi K, Murata K, Sandoval C, Noyori R. The hydrogenation/transfer hydrogenation network: asymmetric hydrogenation of ketones with chiral $\eta 6$-arene/n-tosylethylenediamineruthenium(II) catalysts. JACS.2006;128(27):8724-8725.

32. Pavlova A, Rösler E, Meijer EJ. Mechanistic aspects of using formate as a hydrogen donor in aqueous transfer hydrogenation. ACS Catal. 2016;6(8):5350-5358.

33. Rumble JR. CRC handbook of chemistry and physics (100th edition). CRC Press, 2019.

34. Prego M, Cabeza O, Carballo E, Franjo CF, Jime'nez E. Measurement and interpretation of the electrical conductivity of 1-alcohols from $273 \mathrm{~K}$ to $333 \mathrm{~K}$. J Mol Liq. 2000;89(1):233-238.

35. Prego M, Rilo E, Carballo E, Franjo C, Jiménez E, Cabeza O. Electrical conductivity data of alkanols from 273 to 333 K. J Mol Liq. 2003;102(1):83-91.

36. Smallwood IM. Handbook of organic solvent properties. London:Amold, 1996.

37. Pavlova A, Meijer EJ. Understanding the Role of Water in Aqueous Ruthenium-Catalyzed Transfer Hydrogenation of Ketones. ChemPhysChem. 2012;13(15):3492-3496. 